\title{
Immune checkpoint inhibitor-induced acute tubulointerstitial nephritis
}

\author{
Hui Zhuan Tan ${ }^{1}$, Joycelyn Jie Xin Lee ${ }^{2}$, Chon Jun Jason Choo $^{1}$, Alwin Hwai Liang Loh ${ }^{3}$ \\ ${ }^{1}$ Department of Renal Medicine, Singapore General Hospital, Singapore \\ ${ }^{2}$ Department of Medical Oncology, National Cancer Centre, Singapore \\ ${ }^{3}$ Department of Anatomical Pathology, Singapore General Hospital, Singapore
}

A 69-year-old Chinese man was hospitalized for Common Terminology Criteria for Adverse Events (CTCAE) grade 2 acute kidney injury 8 months following treatment initiation with combination cytotoxic T-lymphocyte-associated protein 4 and programmed cell death protein-1 (PD-1) immune checkpoint blockade for treatment of adenocarcinoma of unknown primary. His medical history included immune checkpoint inhibitor (ICI)-induced adenohypophysitis and gastrointestinal reflux disease, for which proton pump inhibitor (PPI) omeprazole $20 \mathrm{mg}$ twice daily was commenced 6 months prior. His serum creatinine (sCr) peaked at $299 \mu \mathrm{mol} / \mathrm{L}$ from a baseline of $100 \mu \mathrm{mol} / \mathrm{L}$. Urinalysis was notable for pyuria ( 13 white blood cells $/ \mu \mathrm{L}$ ) without hematuria or significant proteinuria (urine protein-creatinine ratio, $0.27 \mathrm{~g} / \mathrm{g}$ ). C-reactive protein was elevated at $51.8 \mathrm{mg} / \mathrm{L}$ (reference range, 0.2-9.1 mg/L). Serum complements were normal. Virology and autoimmune serologies such as hepatitis B, C, anti-double-stranded DNA, and antineutrophil cytoplasmic antibodies were negative. Ultrasonography revealed normal-sized kidneys without obstruction.
A kidney biopsy showed substantial interstitial inflammation and acute tubular injury consistent with ICI-induced acute tubulointerstitial nephritis (ICI-ATIN) (Fig. 1A, B). The diffuse tubulointerstitial mononuclear inflammatory infiltrates comprised lymphocytes, histiocytes, and some plasma cells, without tissue eosinophilia. No interstitial or intratubular granulomas were present. The glomeruli were relatively uninvolved. Interestingly, electron microscopy demonstrated moderate effacement of the foot processes suggestive of podocyte injury (Fig. 1C).

Immunohistochemistry stains showed a predominantly CD8+ lymphocytic infiltrate, macrophages, and scattered plasma cells (Fig. 2). CD3+ T cells constituted about $80 \%$ of the total number of lymphocytes, while CD20+ B cells constituted the remaining $20 \%$. About two-thirds of the T cells were CD8+, while one-third were CD4+. CD68+ histiocytes were fairly abundant. CD138+ plasma cells were the least abundant inflammatory cell type.

Oral prednisolone (PRL) was initiated at $60 \mathrm{mg}(1 \mathrm{mg} / \mathrm{kg})$ daily. Omeprazole was replaced with famotidine, a histamine type-2 receptor antagonist, due to the risk of ICI-ATIN

Received: September 26, 2021; Revised: October 7, 2021; Accepted: October 8, 2021

Correspondence: Tan Hui Zhuan

Department of Renal Medicine, Singapore General Hospital, Academia Level 3, 20 College Road, Singapore 169856, Singapore.

E-mail: tan.hui.zhuan@singhealth.com.sg

ORCID: https://orcid.org/0000-0003-4899-6399

This work was presented, in part, as a "Young Oncologist Patient case studies" oral presentation at the European Society for Medical Oncology (ESMO) Asia Congress 2020. 

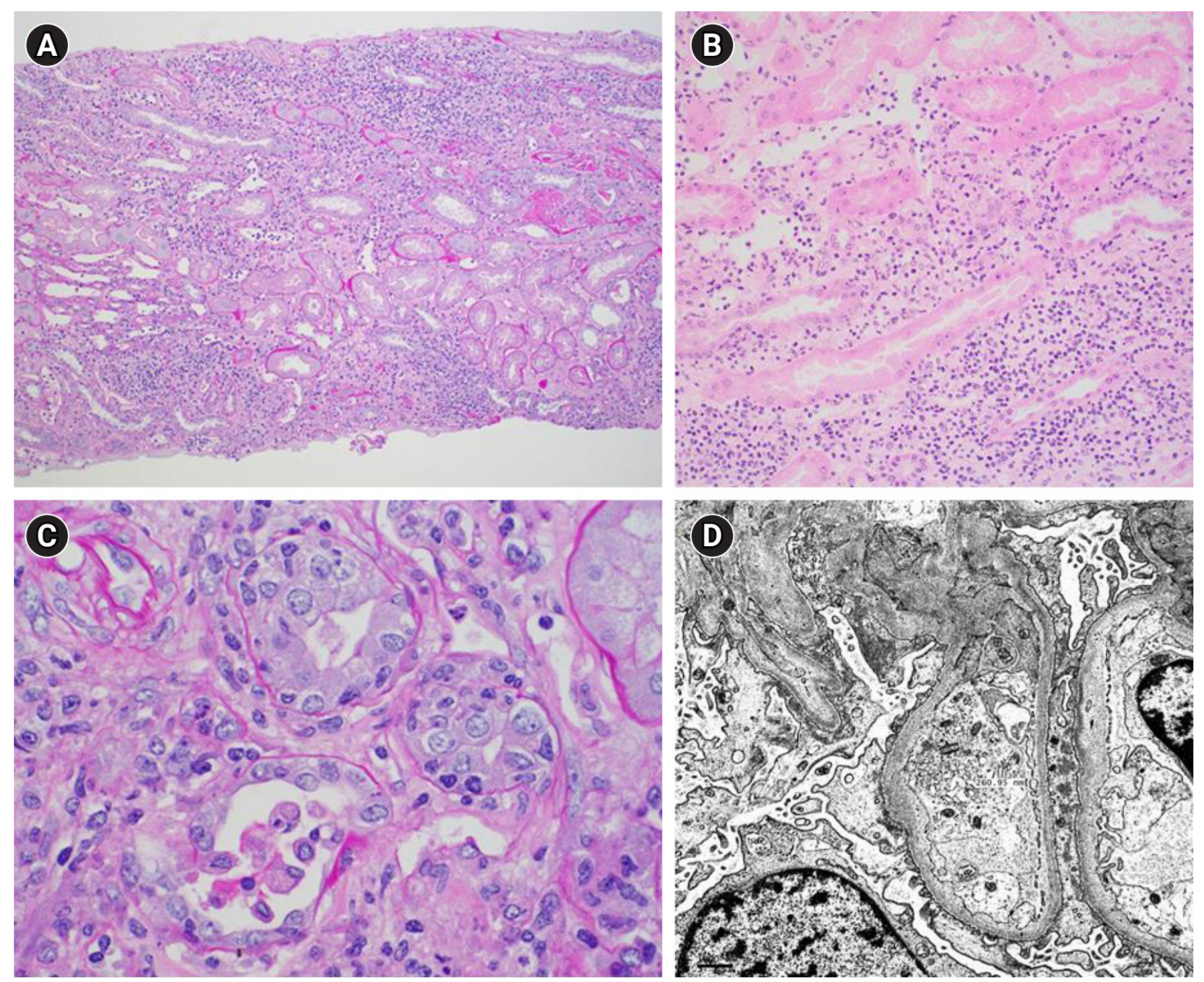

Figure 1. Kidney biopsy showed acute tubulointerstitial nephritis. (A, B) A diffuse tubulointerstitial mononuclear inflammatory infiltrate was noted, comprising lymphocytes, histiocytes, and some plasma cells, without tissue eosinophilia. No well-formed interstitial or intratubular granulomas are present (periodic acid-Schiff, $\times 100$ ). (C) Lymphocytic tubulitis (periodic acid-Schiff, $\times 100)$. (D) Electron microscopy revealed a moderate degree of podocyte foot process effacement, affecting $30 \%$ to $40 \%$ of peripheral capillary wall surface area. This suggests an element of podocyte injury. No electron dense deposits were seen $(\times 6,000)$.

potentiation with the continued use of a PPI. Mycophenolate mofetil (MMF) was added in view of inadequate treatment response and steroid-induced dysglycemia. A repeat biopsy at 6 weeks showed improving interstitial inflammation and marginal worsening of interstitial fibrosis. His $\mathrm{sCr}$ continued to improve, and PRL and MMF were gradually tapered. The single-agent PD-1 ICI was resumed 5 months after the initial ICI-ATIN episode due to rising tumor markers, with PRL maintained at 10mg daily. The sCr remained stable at $140 \mu \mathrm{mol} / \mathrm{L} 10$ months after the ICI rechallenge.

Acute tubulointerstitial inflammation is the most common ICI-related kidney injury, either occurring alone or in combination with other glomerular pathologies [1]. The histopathologic features of ICI-ATIN may resemble acute T cell-mediated renal allograft rejection. Interestingly, electron microscopy of our patient's biopsy revealed subclinical podocyte injury without manifestation of proteinuria. He reported no prior history of diabetes mellitus or use of an- 

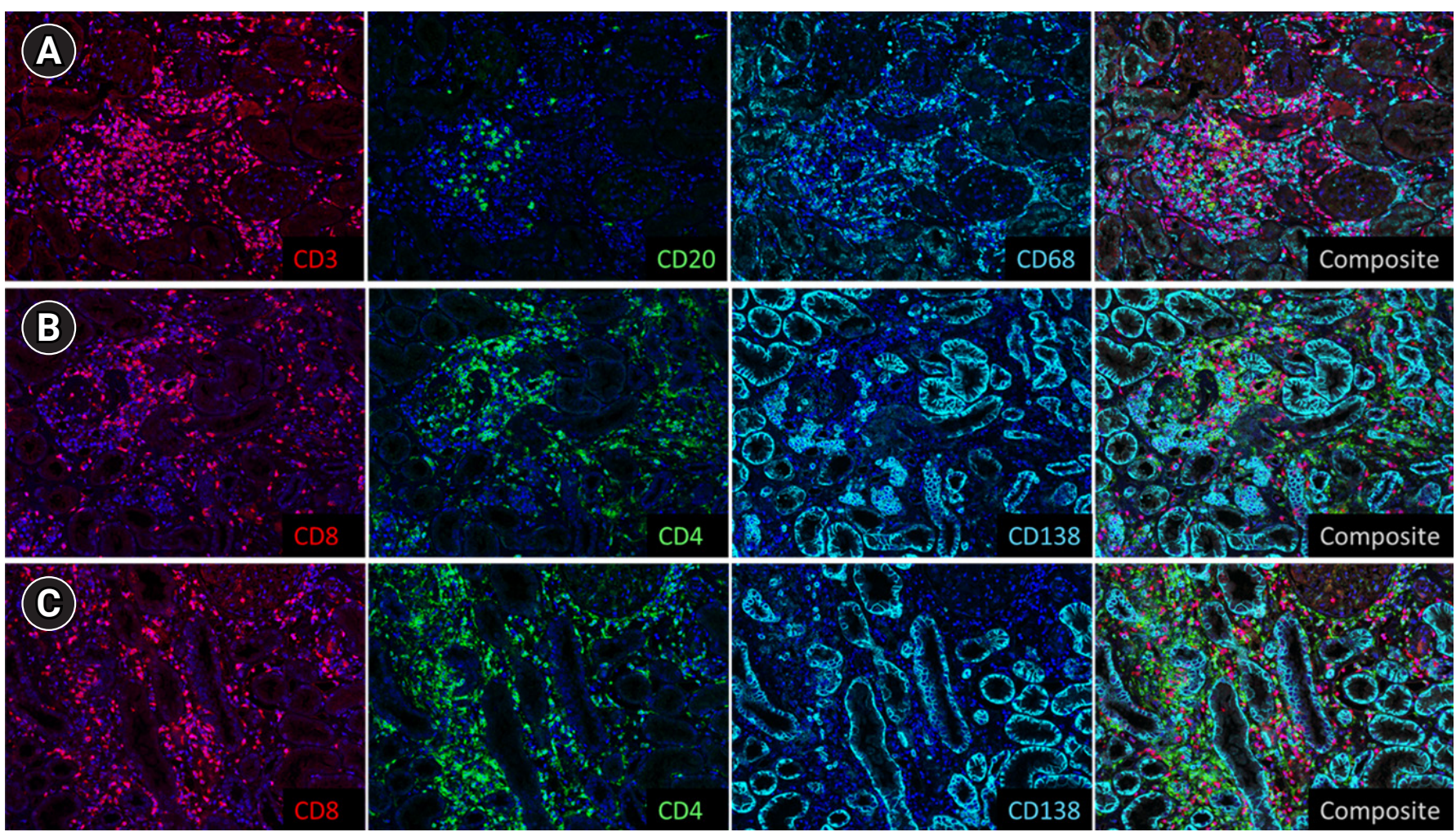

Figure 2. Immunohistochemistry stains showed predominantly CD8+ lymphocytic infiltrate, macrophages, and scattered plasma cells $(\times \mathbf{1 0 0})$. (A) The staining results were analyzed using the Vectra imaging system. CD3+ T cells constituted about $80 \%$ of the total number of lymphocytes, while CD20+ B cells constituted the remaining 20\%. B-lymphocytes formed small aggregates at times. About two-thirds of the T cells were CD8+, while one-third was CD4+. (B, C) Immunohistochemical stains were performed in two different cores of the kidney cortex for better representation. While CD8+ cells were diffusely and evenly distributed throughout the two cores, $\mathrm{CD} 4+$ cells tended to form small aggregates, probably in relation to the presence of B-lymphocytes. Scattered CD138+ plasma cells were observed. Overall, the cell types resembled those seen in acute T cell-mediated renal allograft rejection.

tivascular endothelial growth factor therapies. The clinical significance of this finding is uncertain, but given reports of podocytopathies with ICI use, ongoing monitoring for the development of proteinuria is warranted.

\section{Ethical statements}

In accordance with our institution's research policy, a case report involving one or two patients is not defined as research by the Centralized Institutional Review Board and does not require Centralized Institutional Review Board review, as it does not involve the formulation of a research hypothesis that is subsequently investigated prospectively and systematically for publication or presentation. A letter of support from the Research Office of our institution is pro- vided with the submission of our manuscript. Written informed consent was obtained from the patient with permission to publish the case report and accompanying images.

\section{Conflicts of interest}

Dr. Joycelyn Lee received an honorarium from Ipsen and Bayer and speaker's fees from BMS and Ipsen, and received research funding from Bayer. Associate Professor Jason Chon Jun Choo received consultancy fees from Novartis and was awarded research grants from the National Medical Research Council Singapore, Nikko Dento Corporation, and Singhealth Organization. The remaining authors have no conflicts of interests to report, financial or otherwise. 


\section{Acknowledgments}

We would like to acknowledge Dr. Joe Poh Sheng Yeong for his technical assistance with the images.

\section{Authors' contributions}

Conceptualization: All authors

Writing-original draft: All authors

Writing-review \& editing: All authors

All authors read and approved the final manuscript.

\section{ORCID}

Hui Zhuan Tan, https://orcid.org/0000-0003-4899-6399 Joycelyn Jie Xin Lee, https://orcid.org/0000-0002-1070-6125 Chon Jun Jason Choo, https://orcid.org/0000-0002-8477-0875 Alwin Hwai Liang Loh, https://orcid.org/0000-0003-3852-040X

\section{Reference}

1. Hong MH. Nephrotoxicity of cancer therapeutic drugs: focusing on novel agents. Kidney Res Clin Pract 2021;40:344-354. 